\title{
Calibration of the liquid argon ionization response to low energy electronic and nuclear recoils with DarkSide-50
}

P. Agnes, ${ }^{1}$ I. F. M. Albuquerque, ${ }^{2}$ T. Alexander ${ }^{3}$ A. K. Alton, ${ }^{4}$ M. Ave,${ }^{2}$ H. O. Back, ${ }^{3}$ G. Batignani, ${ }^{5,6}$ K. Biery, ${ }^{7}$ V. Bocci ${ }^{8}$ W. M. Bonivento, ${ }^{9}$ B. Bottino, ${ }^{10,11}$ S. Bussino, ${ }^{12,13}$ M. Cadeddu, ${ }^{9}$ M. Cadoni, ${ }^{14,9}$ F. Calaprice, ${ }^{15}$ A. Caminata,${ }^{11}$ N. Canci, ${ }^{16}$ M. Caravati, ${ }^{9}$ M. Cariello, ${ }^{11}$ M. Carlini, ${ }^{16,17}$ M. Carpinelli, ${ }^{18,19}$ S. Catalanotti, ${ }^{20,21}$ V. Cataudella, ${ }^{20,21}$ P. Cavalcante, ${ }^{22,16}$ S. Cavuoti ${ }^{20,21}$ A. Chepurnov, ${ }^{23}$ C. Cicalò, ${ }^{9}$ A. G. Cocco, ${ }^{21}$ G. Covone,${ }^{20,21}$ D. D’Angelo, ${ }^{24,25}$ S. Davini, ${ }^{11}$ A. De Candia ${ }^{20,21}$ S. De Cecco, ${ }^{8,26}$ G. De Filippis,${ }^{20,21}$ G. De Rosa,${ }^{20,21}$ A. V. Derbin, ${ }^{27}$ A. Devoto, ${ }^{14,9}$ M. D'Incecco, ${ }^{16}$ C. Dionisi, ${ }^{8,26}$ F. Dordei, ${ }^{9}$ M. Downing,${ }^{28}$ D. D’Urso, ${ }^{18,19}$ G. Fiorillo, ${ }^{20,21}$ D. Franco $\odot,{ }^{29}$ F. Gabriele, ${ }^{9}$ C. Galbiati, ${ }^{15,17,16}$ C. Ghiano, ${ }^{16}$ C. Giganti, ${ }^{30}$ G. K. Giovanetti, ${ }^{15}$ O. Gorchakov, ${ }^{31, *}$ A. M. Goretti, ${ }^{16}$ A. Grobov, ${ }^{32,33}$ M. Gromov, ${ }^{23,31}$ M. Guan,${ }^{34}$ Y. Guardincerri, ${ }^{7, *}$ M. Gulino, ${ }^{35,19}$ B. R. Hackett, ${ }^{3}$ K. Herner, ${ }^{7}$ B. Hosseini, ${ }^{9}$ F. Hubaut, ${ }^{36}$ E. V. Hungerford, ${ }^{1}$ An. Ianni, ${ }^{15,16}$ V. Ippolito, ${ }^{8}$ K. Keeter, ${ }^{37}$ C. L. Kendziora, ${ }^{7}$ I. Kochanek, ${ }^{16}$ D. Korablev, ${ }^{31}$ G. Korga, ${ }^{1,16}$ A. Kubankin, ${ }^{38}$ M. Kuss, ${ }^{5}$ M. La Commara, ${ }^{20,21}$ M. Lai ${ }^{14,9}$ X. Li, ${ }^{15}$ M. Lissia, ${ }^{9}$ G. Longo, ${ }^{20,21}$ I. N. Machulin, ${ }^{32,33}$ L. P. Mapelli, ${ }^{39}$ S. M. Mari, ${ }^{12,13}$ J. Maricic, ${ }^{40}$ C. J. Martoff, ${ }^{41}$ A. Messina,,${ }^{8,26}$ P. D. Meyers, ${ }^{15}$ R. Milincic, ${ }^{40}$ M. Morrocchi ${ }^{5,6}$ X. Mougeot, ${ }^{42}$ V. N. Muratova, ${ }^{27}$ P. Musico, ${ }^{11}$ A. Navrer Agasson, ${ }^{30}$ A. O. Nozdrina, ${ }^{32,33}$ A. Oleinik, ${ }^{38}$ F. Ortica, ${ }^{43,44}$ L. Pagani, ${ }^{45}$ M. Pallavicini, ${ }^{10,11}$ L. Pandola, ${ }^{19}$ E. Pantic, ${ }^{45}$ E. Paoloni, ${ }^{5,6}$ K. Pelczar, ${ }^{16,46}$ N. Pelliccia, ${ }^{43,44}$ E. Picciau, ${ }^{14,9}$ A. Pocar, ${ }^{28}$ S. Pordes, ${ }^{7}$ S. S. Poudel, ${ }^{1}$ P. Pralavorio, ${ }^{36}$ F. Ragusa, ${ }^{24,25}$ M. Razeti, ${ }^{9}$ A. Razeto, ${ }^{16}$ A. L. Renshaw, ${ }^{1}$ M. Rescigno, ${ }^{8}$ J. Rode, ${ }^{30,29}$ A. Romani, ${ }^{43,44}$ D. Sablone, ${ }^{15,16}$ O. Samoylov, ${ }^{31}$ W. Sands, ${ }^{15}$ S. Sanfilippo, ${ }^{13,12}$ C. Savarese, ${ }^{17,16,15}$ B. Schlitzer, ${ }^{45}$ D. A. Semenov, ${ }^{27}$ A. Shchagin, ${ }^{38}$ A. Sheshukov, ${ }^{31}$ M. D. Skorokhvatov, ${ }^{32,33}$ O. Smirnov,${ }^{31}$ A. Sotnikov, ${ }^{31}$ S. Stracka, ${ }^{5}$ Y. Suvorov,${ }^{20,21,32}$ R. Tartaglia ${ }^{16}$ G. Testera, ${ }^{11}$ A. Tonazzo, ${ }^{29}$ E. V. Unzhakov, ${ }^{27}$ A. Vishneva, ${ }^{31}$ R. B. Vogelaar, ${ }^{22}$ M. Wada, ${ }^{15,47}$ H. Wang, ${ }^{39}$ Y. Wang, ${ }^{39,34}$ S. Westerdale, ${ }^{15,9}$ M. M. Wojcik, ${ }^{46}$ X. Xiao, ${ }^{39}$ C. Yang, ${ }^{34}$ and G. Zuzel ${ }^{46}$

(DarkSide Collaboration)

\author{
${ }^{1}$ Department of Physics, University of Houston, Houston, Texas 77204, USA \\ ${ }^{2}$ Instituto de Física, Universidade de São Paulo, São Paulo 05508-090, Brazil \\ ${ }^{3}$ Pacific Northwest National Laboratory, Richland, Washington 99352, USA \\ ${ }^{4}$ Physics Department, Augustana University, Sioux Falls, South Dakota 57197, USA \\ ${ }^{5}$ INFN Pisa, Pisa 56127, Italy \\ ${ }^{6}$ Physics Department, Università degli Studi di Pisa, Pisa 56127, Italy \\ ${ }^{7}$ Fermi National Accelerator Laboratory, Batavia, Illinois 60510, USA \\ ${ }^{8}$ INFN Sezione di Roma, Roma 00185, Italy \\ ${ }^{9}$ INFN Cagliari, Cagliari 09042, Italy \\ ${ }^{10}$ Physics Department, Università degli Studi di Genova, Genova 16146, Italy \\ ${ }^{11}$ INFN Genova, Genova 16146, Italy \\ ${ }^{12}$ INFN Roma Tre, Roma 00146, Italy \\ ${ }^{13}$ Mathematics and Physics Department, Università degli Studi Roma Tre, Roma 00146, Italy \\ ${ }^{14}$ Physics Department, Università degli Studi di Cagliari, Cagliari 09042, Italy \\ ${ }^{15}$ Physics Department, Princeton University, Princeton, New Jersey 08544, USA \\ ${ }^{16}$ INFN Laboratori Nazionali del Gran Sasso, Assergi (AQ) 67100, Italy \\ ${ }^{17}$ Gran Sasso Science Institute, L'Aquila 67100, Italy \\ ${ }^{18}$ Chemistry and Pharmacy Department, Università degli Studi di Sassari, Sassari 07100, Italy \\ ${ }^{19}$ INFN Laboratori Nazionali del Sud, Catania 95123, Italy \\ ${ }^{20}$ Physics Department, Università degli Studi “Federico II" di Napoli, Napoli 80126, Italy \\ ${ }^{21}$ INFN Napoli, Napoli 80126, Italy \\ ${ }^{22}$ Virginia Tech, Blacksburg, Virginia 24061, USA \\ ${ }^{23}$ Skobeltsyn Institute of Nuclear Physics, Lomonosov Moscow State University, Moscow 119234, Russia \\ ${ }^{24}$ Physics Department, Università degli Studi di Milano, Milano 20133, Italy \\ ${ }^{25}$ INFN Milano, Milano 20133, Italy \\ ${ }^{26}$ Physics Department, Sapienza Università di Roma, Roma 00185, Italy \\ ${ }^{27}$ Saint Petersburg Nuclear Physics Institute, Gatchina 188350, Russia \\ ${ }^{28}$ Amherst Center for Fundamental Interactions and Physics Department, University of Massachusetts, \\ Amherst, Massachusetts 01003, USA \\ ${ }^{29}$ APC, Université de Paris, CNRS, Astroparticule et Cosmologie, Paris F-75013, France \\ ${ }^{30}$ LPNHE, CNRS/IN2P3, Sorbonne Université, Université Paris Diderot, Paris 75252, France \\ ${ }^{31}$ Joint Institute for Nuclear Research, Dubna 141980, Russia
}




\author{
${ }^{32}$ National Research Centre Kurchatov Institute, Moscow 123182, Russia \\ ${ }^{33}$ National Research Nuclear University MEPhI, Moscow 115409, Russia \\ ${ }^{34}$ Institute of High Energy Physics, Beijing 100049, China \\ ${ }^{35}$ Engineering and Architecture Faculty, Università di Enna Kore, Enna 94100, Italy \\ ${ }^{36}$ Centre de Physique des Particules de Marseille, \\ Aix Marseille Univ, CNRS/IN2P3, CPPM, Marseille, France \\ ${ }^{37}$ School of Natural Sciences, Black Hills State University, Spearfish, South Dakota 57799, USA \\ ${ }^{38}$ Radiation Physics Laboratory, Belgorod National Research University, Belgorod 308007, Russia \\ ${ }^{39}$ Physics and Astronomy Department, University of California, Los Angeles, California 90095, USA \\ ${ }^{40}$ Department of Physics and Astronomy, University of Hawai'i, Honolulu, Hawaii 96822, USA \\ ${ }^{41}$ Physics Department, Temple University, Philadelphia, Pennsylvania 19122, USA \\ ${ }^{42}$ Université Paris-Saclay, CEA, List, Laboratoire National Henri Becquerel (LNE-LNHB), \\ F-91120 Palaiseau, France \\ ${ }^{43}$ Chemistry, Biology and Biotechnology Department, Università degli Studi di Perugia, \\ Perugia 06123, Italy \\ ${ }^{44}$ INFN Perugia, Perugia 06123, Italy \\ ${ }^{45}$ Department of Physics, University of California, Davis, California 95616, USA \\ ${ }^{46}$ M. Smoluchowski Institute of Physics, Jagiellonian University, 30-348 Krakow, Poland \\ ${ }^{47}$ AstroCeNT, Nicolaus Copernicus Astronomical Center, 00-614 Warsaw, Poland
}

(Received 20 July 2021; accepted 15 September 2021; published 8 October 2021)

\begin{abstract}
DarkSide-50 has demonstrated the high potential of dual-phase liquid argon time projection chambers in exploring interactions of WIMPs in the $\mathrm{GeV} / \mathrm{c}^{2}$ mass range. The technique, based on the detection of the ionization signal amplified via electroluminescence in the gas phase, allows us to explore recoil energies down to the sub-keV range. We report here on the DarkSide-50 measurement of the ionization yield of electronic recoils down to $\sim 180 \mathrm{eV}_{\mathrm{er}}$, exploiting ${ }^{37} \mathrm{Ar}$ and ${ }^{39} \mathrm{Ar}$ decays, and extrapolated to a few ionization electrons with the Thomas-Imel box model. Moreover, we present a model-dependent determination of the ionization response to nuclear recoils down to $\sim 500 \mathrm{eV}_{\mathrm{nr}}$, the lowest ever achieved in liquid argon, using in situ neutron calibration sources and external datasets from neutron beam experiments.
\end{abstract}

DOI: 10.1103/PhysRevD.104.082005

\section{INTRODUCTION}

Dual-phase noble liquid time projection chambers (TPCs) have yielded, for more than a decade, ever increasing world leading sensitivity for the search for weakly interacting massive particles (WIMPs) with mass greater than $10 \mathrm{GeV} / \mathrm{c}^{2}$ [1-5]. In recent years, the dual-phase technology has been extended to search for "light" dark matter candidates using the ionization component only, which allows to explore the sub-keV energy range. Experiments like XENON1T with a liquid xenon target [6,7] and DarkSide50 with liquid argon (LAr) [8,9] have set the best limits on WIMP-nucleus interactions for $\mathrm{M}_{\chi}>1.8 \mathrm{GeV} / \mathrm{c}^{2}$ $\left(\mathrm{M}_{\chi}>0.1 \mathrm{GeV} / \mathrm{c}^{2}\right.$ exploiting the Migdal effect), WIMPelectron scattering for $\mathrm{M}_{\chi}>30 \mathrm{MeV} / \mathrm{c}^{2}$, and absorption of dark photons and axionlike particles for $\mathrm{M}_{\chi} \gtrsim 0.2 \mathrm{keV} / \mathrm{c}^{2}$. This was possible thanks to the intrinsic radiopurity and the high ionization yield and resolution, allowing for the detection of single electrons, of noble liquids.

Unlike xenon, for which there exists a rich set of measurements that characterize its ionization response in

\footnotetext{
Deceased.
}

the $\mathrm{keV}$ range (see, for instance. References [10-14]), the argon response is almost unexplored. In spite of the smaller cross section of WIMP scattering on argon compared to xenon, due to the lower atomic number, interactions in argon produce more energetic recoils, with a higher probability of being detected above the threshold. The potential of dualphase LAr TPC technology in direct dark matter search can therefore be significantly enhanced through a better understanding of the ionization response, especially in the sub-keV region. This represents the focus of this paper, through the measurement of the ionization response to electronic (ER) and nuclear (NR) recoils with DarkSide-50, using $\beta$-decay sources intrinsic to $\mathrm{LAr}$, i.e., ${ }^{37} \mathrm{Ar}$ and ${ }^{39} \mathrm{Ar}$, and neutron sources located just outside the TPC, specifically ${ }^{241} \mathrm{Am}-{ }^{13} \mathrm{C}$ and ${ }^{241} \mathrm{Am}-{ }^{11} \mathrm{Be}$. In addition, external datasets from beam test experiments are included in the analysis to better constrain the NR ionization response. This approach, already adopted in the so-called DarkSide-50 low-mass analyses published in 2018 [8,9], is presented in this paper in detail after being highly refined and improved.

Such calibrations not only have the potential to improve current limits with DarkSide-50, but also provide a basis for future detectors specifically designed to search for light dark matter candidates with LAr. 


\section{THE DARKSIDE-50 EXPERIMENT}

The DarkSide-50 experiment is located in Hall C of the Gran Sasso National Laboratory in Italy. The TPC has a cylindrical active LAr target of $\sim 46 \mathrm{~kg}$ The lateral walls are made of polytetrafluoroethylene (PTFE), surrounded by field shaping copper rings. Two arrays of 193 -in diameter photomultiplier tubes (PMTs) are located on both ends behind the transparent anode and cathode, respectively. They observe light signals from both primary scintillation (S1) and electroluminescence (S2) from ionization electrons, extracted in the gas phase, after being drifted with a $200 \mathrm{~V} / \mathrm{cm}$ field in liquid. The extraction efficiency is estimated $>99.9 \%$ at the extraction field of $2.8 \mathrm{kV} / \mathrm{cm}$. The PTFE and the fused silica windows at the top and bottom of the cylinder are coated with tetraphenyl butadiene, a wavelength shifter, that absorbs the $128 \mathrm{~nm}$ photons emission and reemits softer photons with a peak wavelength at $420 \mathrm{~nm}$.

The TPC was first operated, between 2013 and 2014, with atmospheric argon, and then until 2019, with low-radioactivity argon extracted from deep underground, naturally shielded against cosmogenic isotope production. For a more detailed description of the TPC, see Refs. [15,16].

The TPC is surrounded by a hermetic neutron veto, a $4 \mathrm{~m}$ diameter stainless steel sphere filled with $30 \mathrm{t}$ of liquid scintillator, loaded with trimethyl borate molecule at $5 \%$ mass fraction; 110 8-inch PMTs are mounted on the inner surface of the sphere to detect the scintillation light. Neutrons are mostly captured by the ${ }^{10} \mathrm{~B}$ present in the trimethyl borate molecule via the ${ }^{10} \mathrm{~B}(\mathrm{n}, \alpha)^{7} \mathrm{Li}^{*}$ and ${ }^{10} \mathrm{~B}(\mathrm{n}, \alpha)^{7} \mathrm{Li}$ reactions, with a mean capture time of $\sim 22 \mu \mathrm{s}$ [17], thanks to the large

${ }^{10} \mathrm{~B}$ cross section for thermal neutron. The first reaction, with 93.6\% branching ratio, is also responsible for the emission of a characteristic $478 \mathrm{keV} \gamma$ from the ${ }^{7} \mathrm{Li}^{*}$ deexcitation, efficiently detected thanks to the relatively high light yield of the scintillator $(\sim 530 \mathrm{pe} / \mathrm{MeV})$.

The neutron veto is in turn immersed in $1000 \mathrm{t}$ of ultrapure water, instrumented as a Cherenkov veto against cosmic muons, and passive shield against external background. See Ref. [17] for more details on the veto system.

\section{DETECTOR RESPONSE MODEL}

The DarkSide-50 ionization signals are affected by instrumental effects, like the smearing induced by gasphase electroluminescence and by the PMT charge response. The measured $\mathrm{S} 2$ yield $\left(g_{2}\right)$, defined as the mean number of photoelectrons per ionization electron extracted in the gas pocket, and the associated relative resolution are $23 \pm 1 \mathrm{pe} / \mathrm{e}^{-}$and $\sim 27 \%$, respectively, for events localized beneath the central PMT [8]. An additional instrumental effect is related to the electron lifetime, i.e., the survival time of an electron to the capture from impurities in LAr along the drift. This was measured to be about $10 \mathrm{~ms}$, almost 30 times longer than the maximum drift time in the
TPC (376 $\mu \mathrm{s}$ [5]). The distortion of the ionization signal induced by the electron lifetime is therefore limited to a few percent.

The dominant instrumental effect is the dependence of the $\mathrm{S} 2$ response on the event radial position on the $x y$ plane, orthogonal to the electric field. The S2 response yields a factor of $\sim 4$ difference between the center and the edges of the TPC. This has been assessed using the $41.5 \mathrm{keV}$ line from the ${ }^{83 m} \mathrm{Kr}$ gas source injected into the active mass, and applying the position reconstruction algorithm [18], which provides a sub-cm-level resolution. Such a distortion may be due either to a nonuniformity of the tetraphenyl butadiene thickness on the top fused-silica window, which would produce a nonuniform conversion of vacuum ultraviolet scintillation, or to the sagging of the window itself, resulting in a varying thickness of the gas pocket, and, in turn, of the number of electron-luminescence photons produced.

S2 pulses can be corrected using the radial-dependent efficiency measured with ${ }^{83 m} \mathrm{Kr}$ data. However, the energy range of interest for the low-mass analysis extends down to $\sim 100 \mathrm{eV}$, where the reconstruction algorithm is inefficient because of the low number of S2 photoelectrons. The analysis published in 2018 [8] overcame this issue by exploiting a channel-based correction that defines the event position as that of the top-plane PMT $\left(\mathrm{Ch}_{\max }\right)$, which observes the largest fraction of photoelectrons. The position correction based on this definition is rather coarse, because it does not take the finite size (three inches) of the DarkSide-50 PMTs into account.

To improve the accuracy of the measurement of the intrinsic LAr response to ionization signals, all effects mentioned in this section have been incorporated into a Monte Carlo simulation. This approach has been validated on a sample of ${ }^{37} \mathrm{Ar}$ decays, naturally present in LAr. This cosmogenic background decays via a single electron capture transition, ground state to ground state, with a half-life of 35.01 (2) d [19].

The dominant ${ }^{37} \mathrm{Ar}$ decay branches are from the electron capture on $\mathrm{K}(2.83 \mathrm{keV})$ and $\mathrm{L} 1(0.277 \mathrm{keV})$ atomic shells, with branching ratios of $\sim 90.4 \%$ and $\sim 8.4 \%$, respectively, determined with the BETASHAPE code $[20,21]$ using the latest recommended $Q$ value of 813.87 (20) keV [22]. The emitted cascades of electrons, $x$ rays, and UV photons are evaluated with the RELAX software [23], based on the latest EADL2017 library of atomic transition data [24]. RELAX calculates atomic relaxation spectra of UV photons, $x$ rays, and Auger electrons, hereafter referred to as "primaries," due to bound-state-to-bound-state transitions for a single initial vacancy in the different subshells. From the atomic transition probabilities, a deterministic step-by-step propagation of the vacancies was made up to the valence shell and to the neutralization. Atomic configuration has been accounted for, rejecting transitions that would require more electrons in a subshell than those actually present. It should 
TABLE I. Average numbers $(\langle\mathrm{N}\rangle)$ and energies $(\langle\mathrm{E}\rangle)$ of primaries (electrons, $\mathrm{x}$ rays, and UV photons) emitted in electron capture-induced cascades on ${ }^{37} \mathrm{Ar} \mathrm{K}$ and L1 shells as determined using RELAX [23] and the EADL2017 library [24]. The capture probabilities were evaluated with BETASHAPE [20,21]. All energies are expressed in $\mathrm{eV}$.

\begin{tabular}{lcccc}
\hline \hline & \multicolumn{2}{c}{ K-shell EC } & \multicolumn{2}{c}{ L1-shell EC } \\
\hline Branching ratio $^{2} 90.4 \%$ & \multicolumn{2}{c}{$8.4 \%$} & 277 \\
Total released energy & \multicolumn{2}{c}{2829} & \multicolumn{2}{c}{2.8} \\
Mean number of primaries & \multicolumn{2}{c}{3.9} & $\langle\mathrm{~N}\rangle$ & $\langle\mathrm{E}\rangle$ \\
& $\langle\mathrm{N}\rangle$ & $\langle\mathrm{E}\rangle$ & & \\
K Auger electrons & 0.905 & 2414 & & \\
K x-rays & 0.095 & 2634 & & \\
L Auger electrons & 1.77 & 179 & 0.9995 & 179 \\
L X-rays & $8 \mathrm{E}-4$ & 188 & 0.0005 & 207 \\
M Auger electrons & 0.35 & 51 & 0.96 & 51 \\
UV photons (E $>16$ eV) & 0.77 & 25 & 0.86 & 25 \\
Undetectable via ionization & 3.26 & 13 & 2.10 & 13 \\
\hline \hline
\end{tabular}

${ }^{a}$ Excluding undetectable via ionization.

be underlined that the only information available in the EADL2017 library is for a single atomic vacancy. In principle, each additional vacancy due to an Auger transition would require a complete recalculation of the atomic energies, wave functions, and transition probabilities, which is not considered in this work.

The relaxation paths estimated by RELAX are 5213 and 72 for the K and L1 shells, respectively. Each contribution is determined per initial vacancy and weighted by the corresponding capture probability. The average energies of Auger electrons, UV photons, and $\mathrm{x}$ rays grouped by shell, are quoted in Table I. We can assume that the primary particle is almost always an electron, either because directly emitted or because extracted by x rays or UV photons via photoelectric effect. The K and L1 shells emit, on average, 3.9 and 2.8 primaries, respectively, neglecting UV photons with energy not sufficient to photoionize an ${ }^{40} \mathrm{Ar}$ atom. To evaluate the ionization yield, the mean number of primaries will be subtracted from the number of detected electrons, as primaries are also drifted and extracted in the gas pocket and contribute to the $\mathrm{S} 2$ signal.

The two lines from $\mathrm{K}$ and $\mathrm{L} 1$ electron capture are observed in the DarkSide-50 data by subtracting the latest $\sim 500$ days of the underground argon campaign dataset (from 2015 to 2018 ), where ${ }^{37} \mathrm{Ar}$ is almost entirely decayed, from the first $\sim 100$ days, normalizing the two samples by their live times. The subtracted spectrum is fitted with simulated spectra, generated by independently varying the average number of detected electrons $\left(N_{e}\right)$ for each of the two ${ }^{37} \mathrm{Ar}$ lines. Events are simulated with a uniform spatial distribution in the TPC. The intrinsic fluctuations induced by the number of emitted particles and by the LAr ionization response to ERs are modeled with an empirical fudge factor implemented as a Fano factor [25]. In the Monte Carlo data, a fraction of electrons is suppressed according to the electron lifetime. The surviving free electrons are extracted in the gas pocket with $100 \%$ efficiency, and converted into S2 photoelectrons with $g_{2}$ that varies depending on the $x y$ position of the event, according to relative efficiency derived with the ${ }^{83 m} \mathrm{Kr}$ calibration source.

The fit is performed with a $\chi^{2}$ analysis, where the free parameters are the numbers of extracted electrons induced by the two ${ }^{37} \mathrm{Ar}$ lines and the Fano factor. It is worth mentioning that no additional correction is applied to the resolution model. The shoulderlike structure observed at $\sim 35 e^{-}$in Fig. 1 originates from the nonuniform radial response of the detector and is well reproduced by the Monte Carlo data starting from input parameters only.

The resulting numbers of electrons are $12.0 \pm 0.1$ (stat) \pm 0.5 (syst) and $48.2 \pm 0.2$ (stat) \pm 2.1 (syst) for the ${ }^{37} \mathrm{Ar} \mathrm{L1}$ and K shell, respectively. The systematic errors are calculated by propagating the uncertainty on the $g_{2}$ value. The bestfitted simulated spectrum is shown in Fig. 1, together with data, as a function of the reconstructed number of electrons. The fit returns a reduced $\chi^{2}$ of 82.4/64.

The ratio between the measured amplitudes of the two lines, equal to $0.10 \pm 0.01$, is in excellent agreement with $\sim 0.093$, the expected value determined with BETASHAPE, and with measured values in literature $(0.103 \pm 0.003$ [26], $0.102 \pm 0.004$ [27], $0.098 \pm 0.003$ [28]).

The fitted Fano factor, $0.10 \pm 0.03$, is compatible with predictions on the LAr ionization fluctuation from the Shockley (0.107) and the Alkhazov (0.116) models [29]. This result suggests that intrinsic fluctuations of the number of particles emitted in atomic cascades are negligible compared to the ionization fluctuation.

As already mentioned, in order to extract the number of ionization electrons from the two lines, the number of emitted primaries in the atomic cascades must be subtracted from the number of detected electrons. In the L1-shell cascade, the mean number of primaries is 2.8 . The $25 \mathrm{eV}$

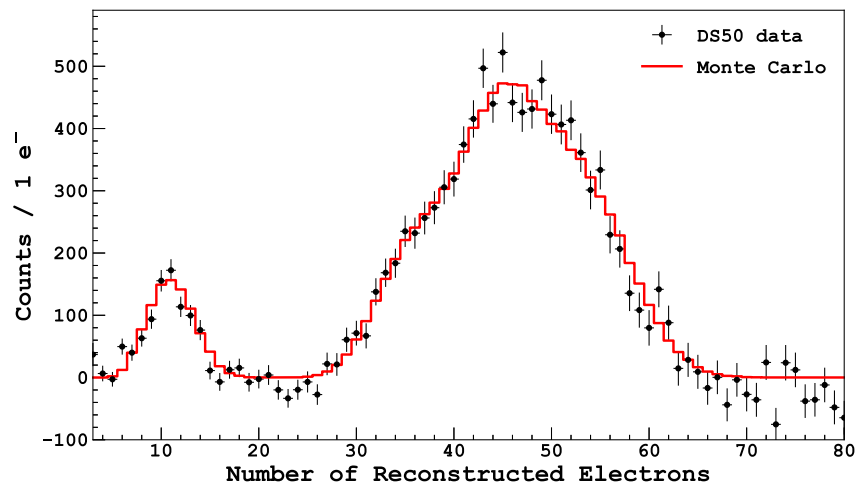

FIG. 1. Comparison between the best-fitted simulated spectrum and ${ }^{37} \mathrm{Ar}$ data as a function of the reconstructed number of electrons. The free parameters in the model are the mean number of ionization electrons induced by the ${ }^{37} \mathrm{Ar} \mathrm{L1-}$ and K-shell electrons, and the Fano factor. 
UV has a high probability of extracting an electron by photoionization but with insufficient energy to induce additional ionization electrons.

The $51 \mathrm{eV}$ M-shell electron is sufficiently energetic to ionize up to two atoms. The Thomas-Imel model, discussed in the following section, predicts a suppressed ion-electron recombination probability at such low ionization densities [30], a finding confirmed by numerical simulations [31]. Yet the energy lost to excitations is unknown at such low energies. Therefore, because of uncertainties on the ionization mechanism, we conservatively assume that the $51 \mathrm{eV}$ line contributes with $1 \pm 1$ ionization electrons to the L1-shell cascade. In addition, we assume negligible interactions between the $51 \mathrm{eV}$ and the L-shell $179 \mathrm{eV}$ electronion clouds because of their low ionization density. The resulting number of ionization electron at $179 \mathrm{eV}$ is $8.2 \pm 1.3$, where the uncertainty takes into account both statistical and systematic errors, corresponding to a ionization yield of $45.7 \pm 7.0 e^{-} / \mathrm{keV}_{\mathrm{er}}$.

Unlike the L1 shell, the superposition of $\sim 3$ ion-electron clouds from K-shell electrons of $2414 \mathrm{eV}$ (or $2634 \mathrm{eV}$ x rays) and $179 \mathrm{eV}$ (1.8 multiplicity) cannot be neglected. However, the lack of a model able to describe the complex event topology does not allow us to estimate the overall recombination effect. For this reason, we do not include data from ${ }^{37} \mathrm{Ar} \mathrm{K}$ shell in the analysis, discussed in the following section, for the determination of the ER ionization yield.

\section{ELECTRONIC RECOIL IONIZATION YIELD}

The calibration of the ER energy scale relies on ${ }^{37} \mathrm{Ar}$ data, discussed in the previous section, and on the cosmogenic ${ }^{39} \mathrm{Ar} \beta^{-}$-decay sample from the 2013-2014 atmospheric argon (AAr) campaign [15], acquired with the same drift field of $200 \mathrm{~V} / \mathrm{cm}$. In AAr, ${ }^{39} \mathrm{Ar}$ has a specific activity of $\sim 1 \mathrm{~Bq} / \mathrm{kg}$ [15], and dominates the event rate. To suppress the "external" background from radioactivity in detector materials surrounding the active mass, events are selected within a central cylinder with $2 \mathrm{~cm}$ radius and $21.6 \mathrm{~cm}$ height, $16.8 \mathrm{~cm}$ far from the lateral walls and $7 \mathrm{~cm}$ from the top and bottom of the TPC. The very narrow cut in radius selects events corresponding to the innermost area of the central PMT only, minimizing the nonuniformity of the detector response. To further remove residual external contamination, events with more than one S2 pulse are rejected. These are multiple scatter events, and not compatible with the topology of the ${ }^{39} \mathrm{Ar} \beta$-decay signature.

The kinetic energy of each event is reconstructed exploiting the full anticorrelation between the S1 and S2 signals, through the so-called rotated energy variable

$$
E_{\mathrm{er}}=w\left(\frac{S 1}{g_{1}}+\frac{S 2}{g_{2}}\right)
$$

where $w=19.5 \pm 1.0 \mathrm{eV}$ [32] is the average energy required to produce a quantum (excitation or ionization) and $g_{1}$ the $\mathrm{S} 1$ collection efficiency $(0.16 \pm 0.01[18])$. The number of ionization electrons escaping the ion-electron recombination process, $N_{\text {i.e. }}$, is calculated for each event of the ${ }^{39} \mathrm{Ar}$ sample as

$$
N_{\text {i.e. }}=\frac{S_{2}}{g_{2}}-1,
$$

which accounts for the subtraction of the primary electron from the $\beta$ decay. The mean value of the ER ionization yield, $Q_{y}^{\mathrm{ER}}$, is estimated using Eqs. (1) and (2) for each $0.2 \mathrm{keV}_{\mathrm{er}}$ bin. A lower threshold of $E_{\mathrm{er}}>1.7 \mathrm{keV}$ is applied to guarantee $100 \%$ efficiency in the identification of the S1 pulse. The uncertainty is dominated by the systematics from the $g_{2}$ parameter.

The ionization yield per unit of ER energy from ${ }^{37} \mathrm{Ar}$ and ${ }^{39} \mathrm{Ar}$ data is defined as

$$
Q_{y}^{\mathrm{ER}}=\frac{N_{\text {i.e. }}}{E_{\text {er }}}=\frac{(1-r) N_{i}}{E_{\mathrm{er}}},
$$

where $N_{i}$ is the number of produced electron-ion pairs. The electron recombination probability $(r)$ is predicted at low energies, $\mathcal{O}\left(\mathrm{keV}_{\mathrm{er}}\right)$, by the Thomas-Imel box model [30],

$$
1-r=\frac{1}{\gamma N_{i}} \ln \left(1+\gamma N_{i}\right)
$$

where $\gamma$ is a free parameter describing the recombination of the initial electron-ion pairs contained in a box and immersed in an electric field. This model has proven to work well in noble liquids for spatially short tracks [33], in the $\mathcal{O}\left(\mathrm{keV}_{\mathrm{er}}\right)$ range for ERs. In this range, Eq. (3) can be parametrized as

$$
Q_{y}^{\mathrm{ER}}=\frac{1}{\gamma} \frac{\ln \left(1+\gamma \rho E_{\mathrm{er}}\right)}{E_{\mathrm{er}}},
$$

with $\rho=N_{i} / E_{\text {er }}$ and $\gamma$ the free parameters of the model. $\gamma$ can be expressed as $C_{\text {box }} / F$, where $F$ is the drift field $(200 \mathrm{~V} / \mathrm{cm})$ and $C_{\text {box }}$ depends on the mean ionization electron velocity and on the size of the ideal box containing the electron-ion cloud. In this parametrization, we assume the approximation of a constant excitation-to-ionization ratio, which implies that $N_{i}$ is proportional to the deposited energy [18]. The impact of this approximation is discussed at the end of this section.

${ }^{39} \mathrm{Ar}$ data, together with the ${ }^{37} \mathrm{Ar}$ calibration line, are fitted with Eq. (5) up to $3 \mathrm{keV}_{\mathrm{er}}$. As shown in Fig. 2, the Thomas-Imel box model is in good agreement with ${ }^{37} \mathrm{Ar}$ and ${ }^{39} \mathrm{Ar}$ data points with $\mathrm{E}_{\mathrm{er}}<3 \mathrm{keV}_{\mathrm{er}}$.

The extension of the model to higher energies through the empirical Doke-Birks parametrization [34], in good agreement with data from the ARIS experiment above $\sim 40 \mathrm{keV}_{\text {er }}$ [35], is not compatible with ${ }^{39} \mathrm{Ar}$ data in the 


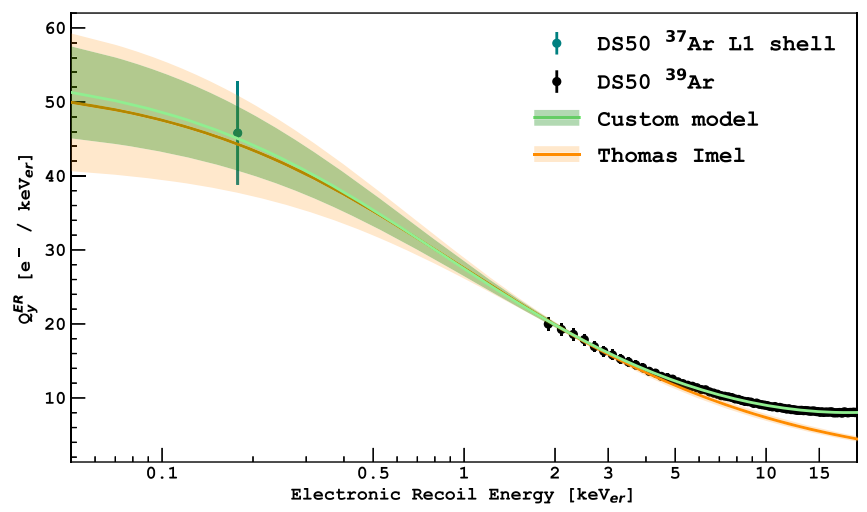

FIG. 2. Fit of the ER ionization yield, measured from AAr (black) and ${ }^{37} \mathrm{Ar}$ (teal) data with a drift field of $200 \mathrm{~V} / \mathrm{cm}$, with the Thomas-Imel box model up to $3 \mathrm{keV}_{\mathrm{er}}$ [Eq. (4)], and with the custom model from Eq. (6), which extends the Thomas-Imel model up to $20 \mathrm{keV}_{\mathrm{er}}$. The model bands correspond to $1 \sigma$, also accounting for the correlation from the $g_{2}$ systematics, which dominates the experimental uncertainties.

3-20 $\mathrm{keV}_{\mathrm{er}}$ range. Instead, the agreement with ${ }^{39} \mathrm{Ar}$ data is recovered in the whole data range, as shown in Fig. 2, by adding a custom term to Eq. (5), as follows

$$
Q_{y}^{\mathrm{ER}}=\left(\frac{1}{\gamma}+p_{0}\left(E_{\mathrm{er}} / \mathrm{keV}_{\mathrm{er}}\right)^{p_{1}}\right) \frac{\ln \left(1+\gamma \rho E_{\mathrm{er}}\right)}{E_{\mathrm{er}}},
$$

with two free parameters, $p_{0}$ and $p_{1}$.

The fit yields $C_{\text {box }}=9.2 \pm 0.9 \mathrm{~V} / \mathrm{cm}, \rho=54.4 \pm$ $7.3 \mathrm{keV}_{\mathrm{er}}^{-1}, p_{0}=0.11 \pm 0.03$, and $p_{1}=1.71 \pm 0.08$. The $C_{\text {box }}$ and $\rho$ parameters are compatible within $1 \sigma$ with $8.6 \pm$ $1.5 \mathrm{~V} / \mathrm{cm}$ and $52.7 \pm 10.9 \mathrm{keV}_{\mathrm{er}}^{-1}$, respectively, obtained from the fit with the Thomas-Imel box model up to $3 \mathrm{keV}_{\mathrm{er}}$. It is worth highlighting that the extrapolation of the ER ionization yield below the lowest measured energy (179 $\mathrm{eV}_{\mathrm{er}}$ from the ${ }^{37} \mathrm{Ar}$ L1-shell electron) is weakly dependent on the custom term introduced in Eq. (6) as it is mainly driven by the Thomas-Imel box model.

To test the impact of the constant excitation-to-ionization ratio assumption, an energy-dependent parametrization was introduced in Eq. (6), so that

$$
\rho \rightarrow \frac{\rho}{1+\alpha\left(E_{\mathrm{er}}\right)},
$$

where

$$
\alpha\left(E_{\mathrm{er}}\right)=\frac{0.21}{1+e^{\left(E_{\mathrm{er}}-b_{1}\right) / b_{2}}}
$$

is a sigmoid function tending to the excitation-to-ionization ratio $\left(\mathrm{N}_{\mathrm{ex}} / \mathrm{Ni}\right)$ of 0.21 , as measured at high energies [34]. The fit of ${ }^{37} \mathrm{Ar}$ and ${ }^{39} \mathrm{Ar}$ data, assuming the change of variable as in Eq. (7), requires two additional free parameters and does not lead to significant variations of $Q_{y}^{\mathrm{ER}}$ with respect to the constant $\rho$ approximation. Therefore, we conclude that data are not sensitive to either assumption and opt to retain the formalism with constant $\mathrm{N}_{\mathrm{ex}} / \mathrm{Ni}$ that represents the simplest model.

\section{NUCLEAR RECOIL IONIZATION YIELD}

The NR ionization yield $\left(Q_{y}^{\mathrm{NR}}\right)$ is formalized in analogy with the ER one in Eq. (3),

$$
Q_{y}^{\mathrm{NR}}=\frac{N_{\text {i.e. }}}{E_{\mathrm{nr}}}=\frac{(1-r) N_{i}}{E_{\mathrm{nr}}},
$$

where the electron recombination probability, $r$, is described by the Thomas-Imel box model in Eq. (4).

Under the assumption that the excitation-to-ionization ratio is constant, $N_{i}$ can be expressed [36] as

$$
N_{i}=\beta \kappa(\epsilon)=\beta \frac{\epsilon s_{e}(\epsilon)}{s_{n}(\epsilon)+s_{e}(\epsilon)},
$$

where $\beta$ is a normalization constant taken as the second free parameter of the model together with $C_{\mathrm{box}}$. The dimensionless parameter $\kappa$ represents the energy lost in electronic excitations giving rise to ionization and scintillation signals. $s_{e}$ is the rate at which electrons are excited by inelastic collisions while $s_{n}$ is the rate at which energy is transferred to recoiling nuclei by elastic collisions. They all depend on the dimensionless parameter $(\epsilon)$ defined as

$$
\epsilon=\frac{a}{2 e^{2} Z^{2}} E_{\mathrm{nr}} / \mathrm{keV} \simeq 0.0135 E_{\mathrm{nr}} / \mathrm{keV},
$$

where the Thomas-Fermi screening length $a=0.626 \cdot a_{0}$. $Z^{-1 / 3}$ [36] is used with $a_{0}=\hbar /\left(\alpha m_{e} c\right) \simeq 0.529 \times 10^{5} \mathrm{fm}$. The stopping power, $s_{e}$, can be expressed as [36]

$$
\begin{aligned}
s_{e}(\epsilon) & =\frac{0.133 Z^{2 / 3}}{A^{1 / 2}} F\left(v / v_{0}\right) \sqrt{\epsilon}, \\
& \simeq 0.145 F\left(v / v_{0}\right) \sqrt{\epsilon},
\end{aligned}
$$

where $F\left(v / v_{0}\right)$ is a correction factor dependent on the nuclear $(v)$ and $\operatorname{Bohr}\left(v_{0}=e^{2} / \hbar\right)$ velocities. With no available theoretical calculation backing either a suppression or an enhancement of the electronic stopping power, we assume $F\left(v / v_{0}\right)=1$. However, at the end of this section, we define an ad hoc function as in Ref. [36] to test the sensitivity of the calibration data to a potential suppression of $s_{e}(\epsilon)$ at low energies.

The nuclear stopping power, $s_{n}$, is modeled by Ziegler et al. using an universal screening function [37]:

$s_{n}(\epsilon)=\frac{\ln \left(1+1.1383 f_{Z} \epsilon\right)}{2\left[f_{Z} \epsilon+0.01321\left(f_{Z} \epsilon\right)^{0.21226}+0.19593\left(f_{Z} \epsilon\right)^{0.5}\right]}$, 
where $f_{Z} \simeq 0.953$ is a conversion factor for argon that accounts for the slightly different dimensionless energy definition used in Ref. [37] compared to Eq. (11).

The NR response model is applied to simulated events analogously to what done for ERs in Sec. III. The only difference is in the intrinsic resolution of the NR ionization process. Following the procedure used in Ref. [8], we considered two extreme models: one allowing for fluctuations in energy quenching, ionization yield, and recombination processes obtained with binomial distributions, and an other where the fluctuations in energy quenching are set to zero. The analysis described in the below cannot distinguish between the two models, as the difference in the results is negligible. For this reason, in the following, we only consider the model without quenching fluctuations.

The model from Eq. (9) is constrained by fitting DarkSide50 calibration data, using ${ }^{241} \mathrm{Am}^{-13} \mathrm{C}$ and ${ }^{241} \mathrm{Am}-{ }^{9} \mathrm{Be}$ neutron sources [38], acquired during the underground argon (UAr) campaign, and external datasets from the SCENE [39], ARIS [35], and Joshi et al. [40] experiments, as described in the following.

\section{A. ${ }^{241} \mathrm{Am}-{ }^{13} \mathrm{C}$ data selection}

The ${ }^{241} \mathrm{Am}-{ }^{13} \mathrm{C}$ (from now on $\mathrm{AmC}$ ) neutron source [41] is located outside the DarkSide-50 cryostat, in the liquid scintillator veto, as shown in Fig. 3. The source emits neutrons via $(\alpha, \mathrm{n})$ on ${ }^{13} \mathrm{C}$, producing ${ }^{16} \mathrm{O}$ in the ground level in the final state. First or second excited states,

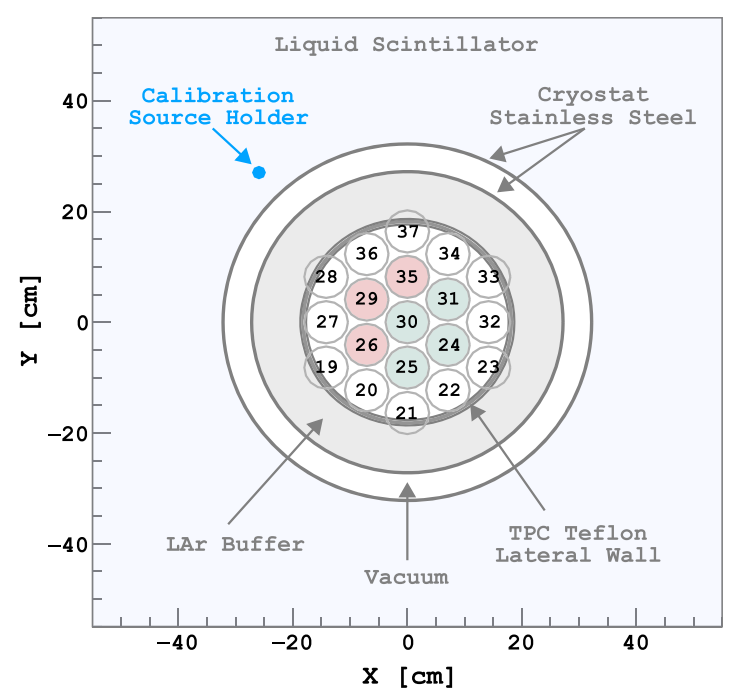

FIG. 3. Schematic top view of the detector. The source holder is located in the liquid scintillator veto. Source events have to cross the cryostat, the LAr buffer and the PTFE walls before reaching the active mass. Events whose position is associated to channels in the outer ring (white) are not included in this analysis. Only events with maximum fraction of light observed by PMTs highlighted in green in the figure are included in the AmC analysis. The analysis of the AmBe source is extended also to events selected by PMTs highlighted in red. accompanied by $\gamma$ emission from ${ }^{16} \mathrm{O}^{*}$ deexcitation, are suppressed by a thin degrader, which reduces the $\alpha$ energy below that needed to reach the lowest excited state of ${ }^{16} \mathrm{O}$.

A $2 \mathrm{~mm}$ thick lead shielding absorbs ${ }^{241} \mathrm{Am} \times$ rays, resulting in a neutron source with very low correlation with $\gamma$ and $\mathrm{x}$ emission. However, to compensate for the low efficiency in neutron production, the ${ }^{241} \mathrm{Am}$ activity is rather high $(\sim 3.6 \mathrm{MBq})$, producing pile-up $\mathrm{x}$ rays and $\gamma \mathrm{s}$ with a non-negligible probability of escaping the shielding and reaching the active volume of DarkSide-50, crossing the cryostat, the LAr buffer surrounding the TPC, the fieldshaping copper rings, and the PTFE walls housing the active volume.

The ${ }^{241} \mathrm{Am} \gamma$ ray with the highest branching ratio (35.9\%) has an energy of $59.5 \mathrm{keV}$ that falls in the regime dominated by the photoelectric effect and is fully absorbed in the LAr buffer and preceding materials. ${ }^{241} \mathrm{Am} \gamma$ rays with an energy $>99 \mathrm{keV}$ and branching ratio $>10^{-9}$, as quoted in [42], are simulated with G4DS [18], the DarkSide-50 Monte Carlo simulation package or Monte Carlo simulation code, to derive the spectrum of AmC uncorrelated events. The detector response is applied as described in Sec. III.

The AmC NR analysis is performed on events with $\mathrm{Ch}_{\max }$ corresponding to channels $24,25,30$, and 31 , the four central PMTs less exposed to the source, as shown in Fig. 3, with ${ }^{241} \mathrm{Am} \gamma$ contamination minimized to $5.2 \%$, as estimated from a Monte Carlo simulation. Events selected by channels 26, 29, and 35 (see Fig. 3) are excluded from the analysis because of the high rate of ${ }^{241} \mathrm{Am} \gamma \mathrm{s}$. Events not correlated with the source are subtracted using data from the UAr campaign, selected with the same $\mathrm{Ch}_{\max }$ cuts and normalized by the live time.

Figure 4 shows AmC and UAr data spectra, the latter normalized by the live time, and the simulated spectrum of $\gamma$ events from ${ }^{241} \mathrm{Am}$ reaching the TPC. The uncertainty on the source position affects the amplitude of the simulated spectrum, which is then normalized to the UAr-subtracted

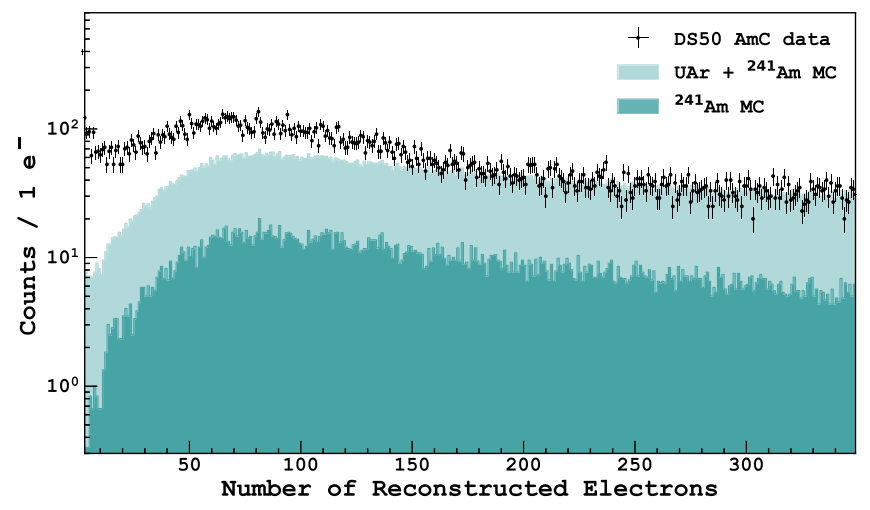

FIG. 4. Comparison between AmC spectrum (black) and contamination from TPC intrinsic events and $\gamma \mathrm{s}$ from ${ }^{241} \mathrm{Am}$, as described in the text. The low-energy excess is due nuclear recoils induced by neutrons. 
AmC spectrum in the $[250,900] N_{e}$ range, where no NR event is expected. The excess of events from the source, shown in Fig. 4, with respect to the TPC intrinsic contamination and to simulated ${ }^{241} \mathrm{Am} \gamma$ rays, is attributed to neutron scatterings.

\section{B. ${ }^{241} \mathrm{Am}-{ }^{9} \mathrm{Be}$ data selection}

The ${ }^{241} \mathrm{Am}-{ }^{9} \mathrm{Be}$ source (from now on $\mathrm{AmBe}$ ) emits neutrons in association with the emission of, among others, 4.4 MeV $\gamma \mathrm{s}$. The prompt $\gamma$ signal is detected in the liquid scintillator veto [17], where the source is deployed. Most neutrons, once scattered in the TPC LAr target, escape the TPC given the low neutron capture cross section in ${ }^{40} \mathrm{Ar}$. Scattered neutrons that reach the neutron veto are mostly captured by ${ }^{10} \mathrm{~B}$ with $22 \mu$ s mean time.

NRs in the TPC are selected with a threefold coincidence by looking at the prompt $4.4 \mathrm{MeV} \gamma$, followed by a singlescatter signal in the TPC within a few tens of nanoseconds, in turn followed by the delayed neutron capture in the veto. In order to apply this selection, events with both $\mathrm{S} 1$ and $\mathrm{S} 2$ pulses are required. The events with $\mathrm{S} 2$ signal only, in fact, are delayed by the drift time up to a maximum of $376 \mu$ s, much longer than the coincidence time with the prompt signal in the veto, which, in most cases, falls outside the acquisition window. On the contrary, the $\mathrm{S} 1$ signal is very fast, and allows us to not miss the coincidence with the prompt signal in the veto. The requirement of an S1 signal, however, implies an energy threshold, dictated by the $\mathrm{S} 1$ trigger and pulse-finder efficiencies. The overall efficiency for NR events is defined as the ratio between two-pulses events $(\mathrm{S} 1+\mathrm{S} 2)$ and all events with either $\mathrm{S} 2$ only or $\mathrm{S} 1+\mathrm{S} 2$. The efficiency is extracted from the AmC dataset, by subtracting the ER contamination from each of the two S2-only and S1 + S2 samples. The resulting efficiency, which from now on is named S1 detection efficiency, is fitted with an error function, which takes into account

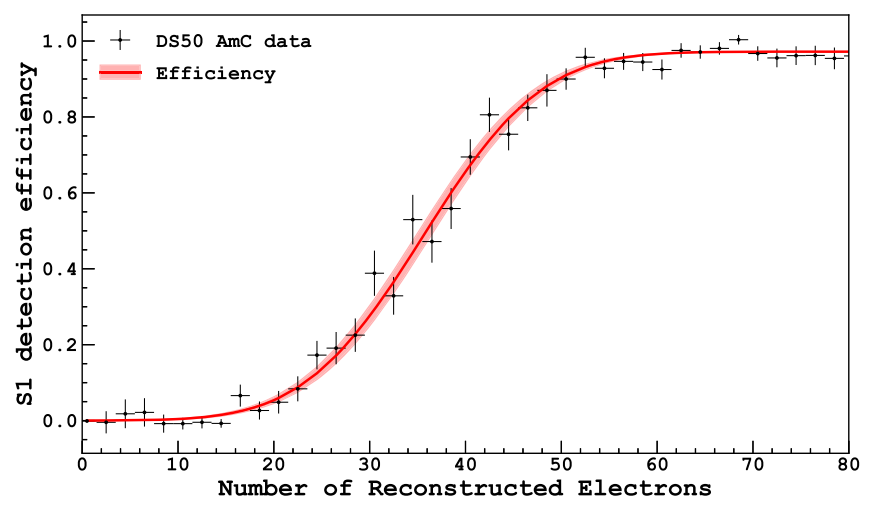

FIG. 5. Efficiency in detecting $\mathrm{S} 1+\mathrm{S} 2$ events $\left(\mathrm{N}_{2 p}\right)$ with respect to $\mathrm{S} 1+\mathrm{S} 2$ and $\mathrm{S} 2$ only $\left(\mathrm{N}_{1 p}\right)$, as a function of the reconstructed number of electrons. Data are fitted with an error function (red line). The error band is propagated from the uncertainties of the fitted parameters.
Gaussian fluctuations expected in the S1 efficiency, as shown in Fig. 5.

Prompt signals in the veto are selected by requiring an electron equivalent energy in the $[4,5] \mathrm{MeV}$ range, and a time difference with respect to the TPC between -600 and $-500 \mathrm{~ns}$, taking into account the time offset of $-550 \mathrm{~ns}$ between veto and TPC. The delayed signal is selected in the $[10,45] \mu$ s time window, and with event energy in the [370, $510] \mathrm{keV}$ range, a range properly tuned to account for quenching effects and $\gamma$ energy deposition in passive materials. The $10 \mu \mathrm{s}$ low time threshold is applied to avoid veto PMT afterpulses. The selection cuts are shown in Fig. 6.

The AmBe events selected in triple coincidence still suffer from a residual contamination due to accidental ERs. Such a contamination is account for by looking in control region where no NR is expected. The control region is defined selecting events with $N_{e}$ in the $[200,500]$ range and with $f_{90}<0.4$, where $f_{90}$ is the pulse shape discrimination estimator of DarkSide-50 [15], less than 0.4, a region where no NR is expected. The UAr spectrum is normalized with respect to the same selection criteria, and subtracted from the $\mathrm{AmBe}$ one. The contributions from $\mathrm{AmBe}$ and nonsource events are shown in Fig. 7.

\section{External datasets}

A further constraint to the LAr response to the ionization signal is provided by "external" datasets, i.e., measurements performed with small scale LAr detectors exposed to neutron beams. The SCENE collaboration [39] measured the ionization yield for four NR energies, between 16.9 and $57.3 \mathrm{keV}$, with $g_{2}=3.1 \pm 0.3 \mathrm{pe} / \mathrm{e}^{-}$. The drift field at $193 \mathrm{~V} / \mathrm{cm}$ is very close to one used in DarkSide-50 $(200 \mathrm{~V} / \mathrm{cm})$, and the difference is assumed negligible in this analysis. SCENE results are normalized to the DarkSide-50 response by the ratio between the corresponding $g_{2} \mathrm{~s}$.

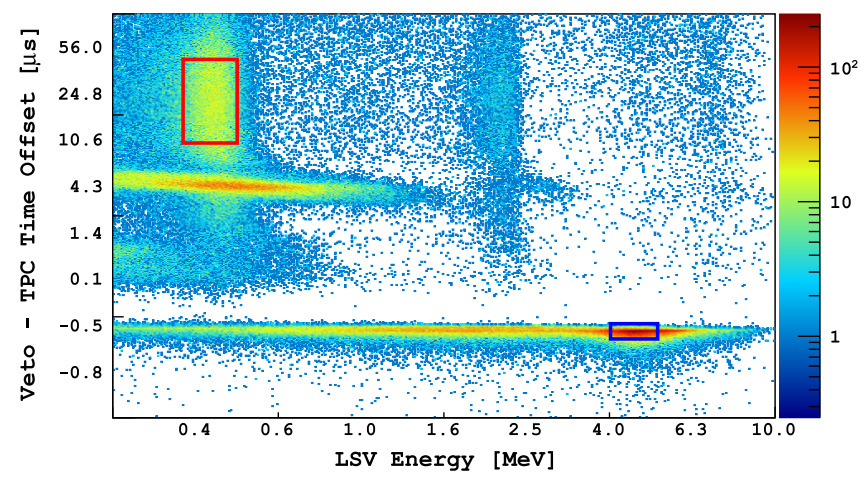

FIG. 6. Veto selection of prompt (blue box) and delayed (red box) events associated to AmBe neutron emission, as a function of liquid scintillator veto (LSV) visible energy and time difference between veto and TPC signals. The trigger time offset between the two detectors is about $-550 \mathrm{~ns}$. The horizontal feature at the $4.3 \mu$ s offset is due to PMT afterpulses. 


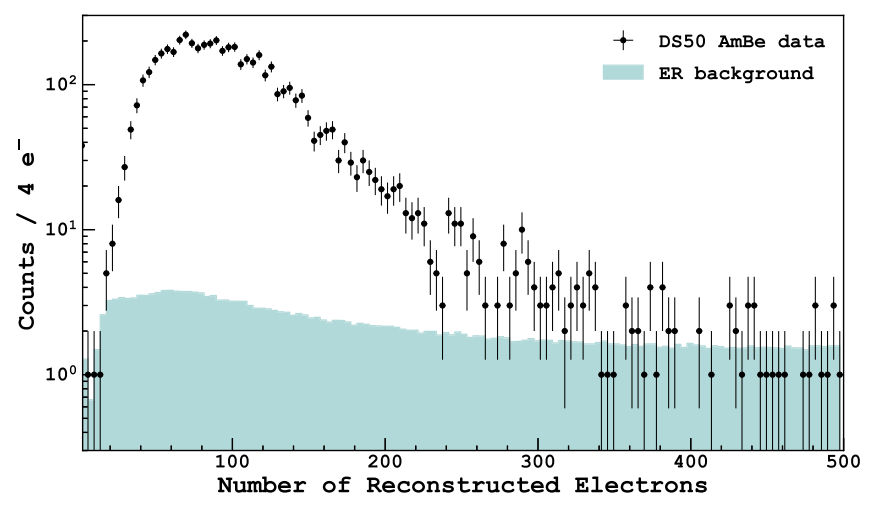

FIG. 7. Spectrum of events selected in triple coincidence (black), compared with accidentals with $f_{90}<0.4$ from the UAr sample, normalized to events selected in the AmBe sample with $N_{e}$ in the $[200,500]$ range.

The ARIS collaboration [35] characterized the LAr scintillation response at $200 \mathrm{~V} / \mathrm{cm}$ for eight NR energies, between 7.1 and $117.8 \mathrm{keV}$. ARIS S1 data at $200 \mathrm{~V} / \mathrm{cm}$ are rescaled to the DarkSide-50 response by the ratio between field-off S1 yields of DarkSide-50 $(8.0 \pm 0.1 \mathrm{pe} / \mathrm{keV})$ and ARIS $(6.35 \pm 0.05 \mathrm{pe} / \mathrm{keV})$. This allows us to associate the NR nominal energies from ARIS to the correspondent $\mathrm{S} 1$ at $200 \mathrm{~V} / \mathrm{cm}$ in DarkSide-50. The final step is the conversion of the so-obtained $\mathrm{S} 1$ values to $\mathrm{S} 2$, by looking at the S2/S1 ratio from NRs selected with the triple coincidence in the AmBe dataset. The correlation between $\mathrm{S} 1$ and S2 signals for the AmBe source was accounted for by means of Monte Carlo simulation, which embeds the DarkSide-50 energy and optical response models [18]. The resulting ionization yield is slightly lower at low energies than that published in 2018 [8]. This difference is due to the improved modeling of the detector response.

Joshi et al. [40] have measured the ionization yield of NRs at $6.7 \mathrm{keV}$ using the end point of a spectrum induced by monochromatic $70 \mathrm{keV}$ neutrons from a beam. The measurement at $240 \mathrm{~V} / \mathrm{cm}$, the closest field to the DarkSide-50 one, results in $\mathrm{Q}_{y}=3.6_{-1.1}^{+0.5} \mathrm{e}^{-} / \mathrm{keV}$. However, after consulting with the authors, the data point is corrected for their single electron yield using the $2.82 \mathrm{keV} \mathrm{K}$-shell capture ${ }^{37} \mathrm{Ar}$ line from their experiment and DarkSide-50 as a cross-calibration point. The corrected value is then $\mathrm{Q}_{y}=6.0_{-1.8}^{+0.8} \mathrm{e}^{-} / \mathrm{keV}$. However, because of this correction, we preferred not to include the measurement by Joshi et al. in this analysis but to quote it in the comparison with the final result.

\section{Global fit to data}

The energy Monte Carlo spectra for fitting both AmC and $\mathrm{AmBe}$ data samples are generated with G4DS, which accurately describes detector and source geometries, with a statistics of about $10^{5}$ neutron events, between 1 and 2 orders of magnitude higher than the data statistics.
The detector response is applied with a toy Monte Carlo approach, as described earlier, by varying $\beta$ and $C_{\text {box }}$ parameters from the model with a fine scan. In addition, in order to take into account the inefficiency on S1 pulses, the efficiency curve shown in Fig. 5 is applied to AmBe simulated events as a function of $N_{e}$.

The AmC and AmBe datasets are simultaneously fitted with a $\chi^{2}$ analysis in the $[3,250] N_{e}$ range. The fit includes $g_{2}$ as an additional fit parameter constrained to the measured value within its uncertainty. To minimize the statistical fluctuations, the $\chi^{2}$ value is averaged over 800 toy simulations for each $\left(\beta, C_{\mathrm{box}}\right)$ parameter pair. The resulting $\chi^{2}$ map in the $\left(\beta, C_{\mathrm{box}}\right)$ parameter space is summed to the one from the simultaneous fit of the external datasets from SCENE and ARIS presented in the previous section.

The resulting best parameters of such a global fit are $C_{\text {box }}=8.1_{-0.2}^{+0.1} \mathrm{~V} / \mathrm{cm}$ and $\beta=\left(6.8_{-0.3}^{+0.1}\right) \times 10^{3}$. The corresponding model of the ionization yield is shown as a function of the nuclear recoil energy in Fig. 8. The errors on the model are from statistical uncertainties and systematic error on $g_{2}$. The $Q_{y}^{\mathrm{NR}}$ extracted from external datasets only is in excellent agreement with the one from AmC and AmBe calibrations, as shown in Fig. 8, and the reduced $\chi^{2}$ from combined fit of all the datasets is equal to 1.34 $\left(\chi^{2} / \mathrm{NDF}=676 / 506\right)$. Figure 9 shows the comparison between data and model, for both $\mathrm{AmC}$ and $\mathrm{AmBe}$, assuming best-fitted parameters. The model is constrained at low energy by the AmC calibration data, with a minimum energy value of $435_{-34}^{+47} \mathrm{eV}_{\mathrm{nr}}$, corresponding to three electrons. This is the lowest NR calibration threshold ever achieved in LAr.

Regarding potential systematics, we have investigated the impact of S1 detection efficiency on the result for five low thresholds, ranging from 0 to $40 N_{e}$, applied to the $\mathrm{AmBe}$ dataset. The results obtained by repeating the fit for

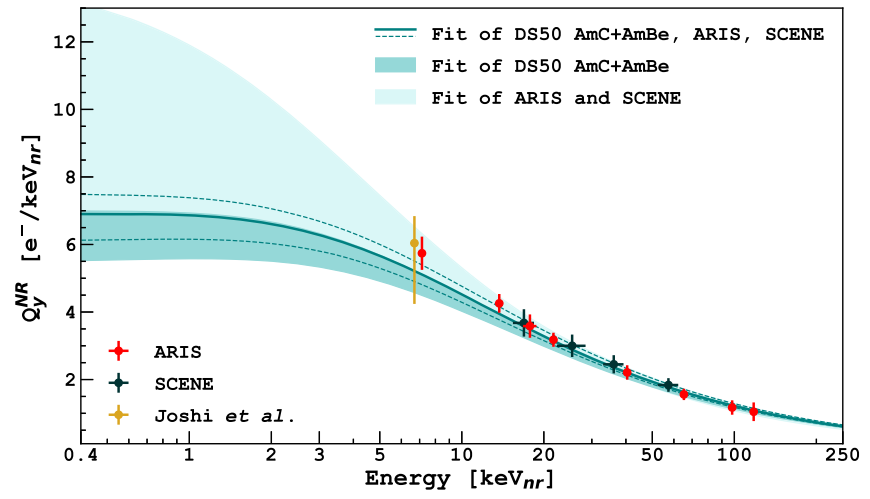

FIG. 8. Fit of the NR ionization yield at $200 \mathrm{~V} / \mathrm{cm}$ from the combined fit of DarkSide-50 AmBe and AmC calibration data, together with datasets from SCENE [39] and ARIS measurements [35], the latter combined with the DarkSide-50 ionizationto-scintillation ratio. The measured $Q_{y}^{\mathrm{NR}}$ by Joshi et al. [40] at $6.7 \mathrm{keV}_{\mathrm{nr}}$ is reported for comparison. The model bands correspond to $1 \sigma$ uncertainty. 

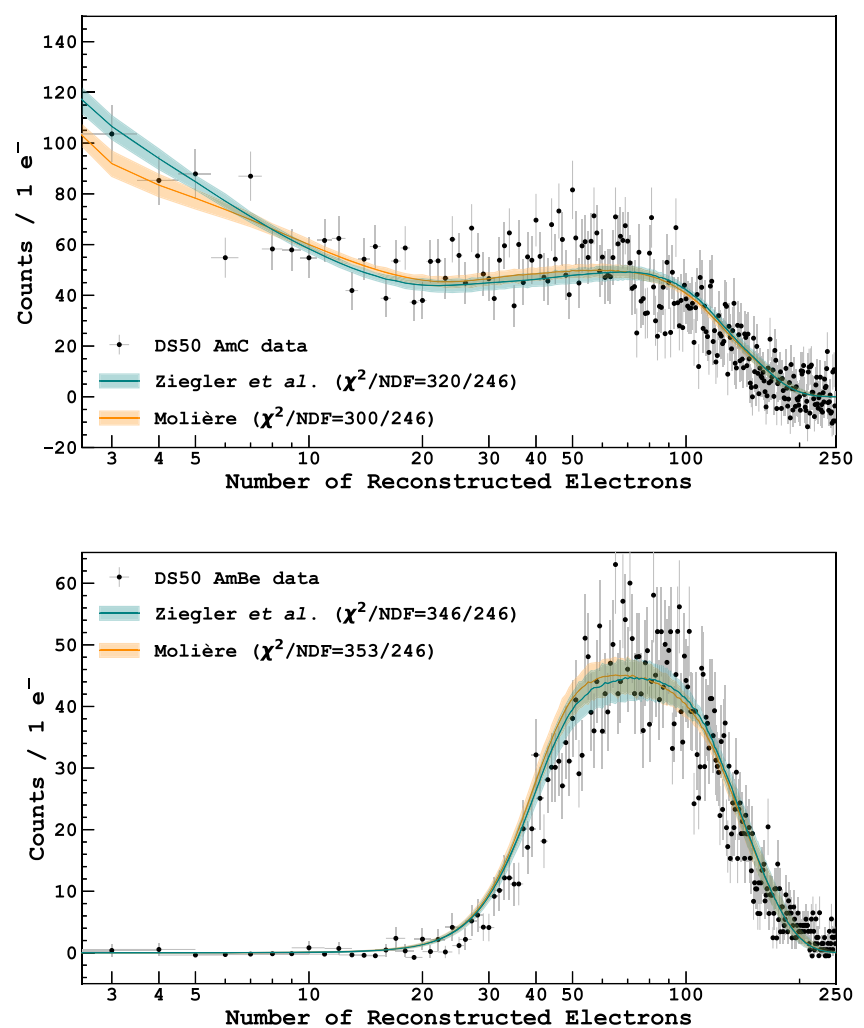

FIG. 9. Best fitted simulated $N_{e}$ spectra using Ziegler et al. (blue) and Molière (orange) screening functions, compared to AmC (top) and AmBe (bottom) data. The model bands correspond to $1 \sigma$ uncertainty.

each threshold are in agreement within $1 \sigma$ with the one without threshold, confirming that the S1 detection efficiency does not induce any appreciable systematics.

\section{E. Sensitivity to other theoretical models}

We have investigated the sensitivity of our data to different models of the nuclear stopping power as a function of energy stemming from different assumption on screening effects from atomic electrons. This is encapsulated in screening functions arising from different models that can be written as [36]

$$
f(\eta)=\frac{\lambda \eta^{1-2 m}}{\left(1+\left[2 \lambda \eta^{2(1-m)}\right]^{q}\right)^{1 / q}},
$$

with each model characterized by a different set of parameters. The additional tested models, as suggested by Bezrukov et al. [36], are Molière $(m=0.216, q=0.570$, $\lambda=2.37)$ [43], and Lenz-Jensen $(m=0.191, q=0.512$, $\lambda=2.92)[44,45]$. Each of them leads to a different nuclear stopping power through

$$
s_{n}(\epsilon)=\frac{1}{\epsilon} \int_{0}^{\epsilon} f(\eta) d \eta,
$$

with $\epsilon$ from Eq. (11). The Thomas-Fermi screening function, also investigated by Bezrukov et al. [36], is not included in

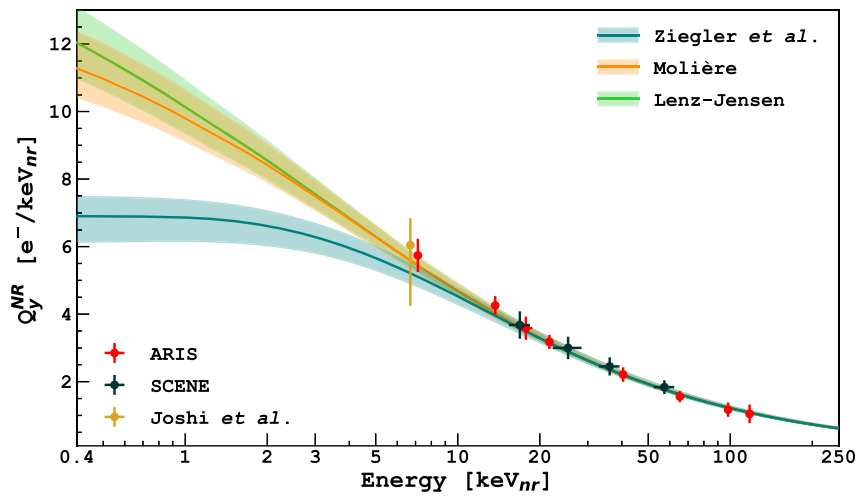

FIG. 10. Fit of DarkSide-50 AmBe and AmC data, and ARIS and SCENE datasets using Ziegler et al. [Eq. (13)] [37], Molière [43], and Lenz-Jensen [44,45] screening functions. The model bands correspond to $1 \sigma$ uncertainty.

this study as it is relevant when the projectile is a naked nucleus or an elementary particle but not for partially ionized atoms.

The above-described analysis was performed for the Molière and Lenz-Jensen models, and it was found that both can fit successfully the data, with no statistically significant difference among them. The $Q_{y}^{\mathrm{NR}} \mathrm{S}$ obtained from the fit using the different screening functions are shown in Fig. 10. The comparison between model and data for two cases, Ziegler et al. and Molière, is shown in Fig. 9.

The Ziegler et al. model is the one yielding the lowest $Q_{y}^{\mathrm{NR}}$ in the region of interest for WIMP analysis. Therefore the adoption of this model will result in the more conservative choice for the sensitivity to WIMPs in future DarkSide searches.

Following the same conservative approach, we also explored the impact of a low-energy $s_{e}$ suppression by introducing the functional form of $F\left(v / v_{0}\right)$ in Eq. (12) as suggested in [36]:

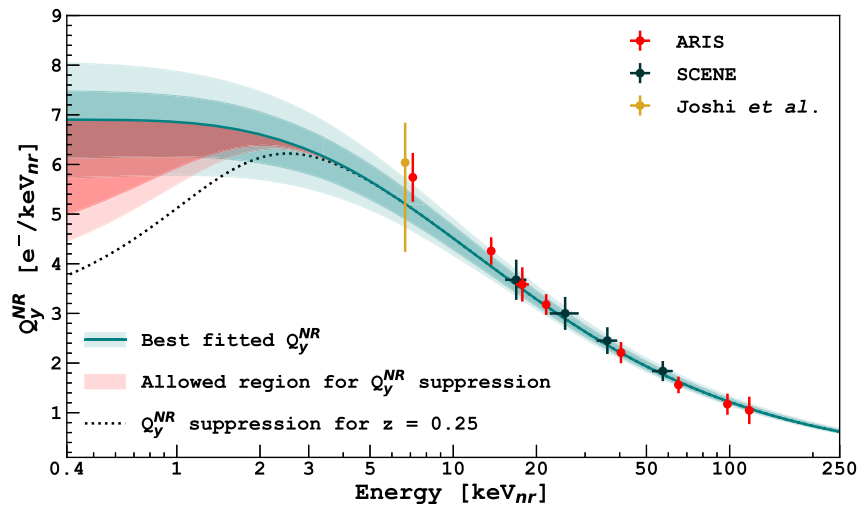

FIG. 11. Allowed region (red) for low energy $Q_{y}^{\mathrm{NR}}$ suppression tested on the AmC dataset, and best-fitted $Q_{y}^{\mathrm{NR}}$ (blue), using the Ziegler et al. screening function. Dark (light) shaded area corresponds to $1 \sigma(2 \sigma)$ uncertainty. The dashed line represents the $Q_{y}^{\mathrm{NR}}$ suppression tested in [36] assuming Eq. (16) with $z=0.25$. 


$$
F\left(v / v_{0}\right)=1 / 2(1+\tanh (50 \epsilon-z)),
$$

where $F\left(v / v_{0}\right) \rightarrow 1$ for $z \rightarrow-\infty$. Setting $z=0.25$ enables us to reproduce the attempt in [46] to include Coulomb effects in the calculation of the electronic stopping power. Such a suppression, demonstrated to be not compatible with existing LXe datasets [36], affects the energy range in LAr below $\sim 3 \mathrm{keV}_{\mathrm{nr}}$, as shown in Fig. 11. Through a null hypothesis test, where the null hypothesis corresponds to $F\left(v / v_{0}\right)=1$, we verified that low energy AmC data have the power to constrain suppression effect to be within $\sim 18 \%(2 \sigma)$ at $1 \mathrm{keV}_{\mathrm{nr}}$ below the nominal charge yield derived from the universal function. This test allows us to exclude $z>0.04$ or $Q_{y}^{\mathrm{NR}}\left(1 \mathrm{keV}_{\mathrm{nr}}\right)<5.6 e^{-} / \mathrm{keV}_{\mathrm{nr}}$ at $95 \%$ C.L.

\section{CONCLUSIONS}

In this work, we presented the calibration of the LAr ionization response to electronic and nuclear recoils in the $\mathrm{keV}$ region at $200 \mathrm{~V} / \mathrm{cm}$ with DarkSide-50. The electronic recoil one is measured down to $179 \mathrm{eV}_{\mathrm{er}}$, the energy of the L1-shell Auger electron from ${ }^{37} \mathrm{Ar}$, and extrapolated down to few tens of eV by fitting data with the Thomas-Imel box model. The nuclear recoil ionization response is measured with a low threshold of $\sim 500 \mathrm{eV}_{\mathrm{nr}}$, corresponding to three ionization electrons, the lowest ever performed in LAr. The measured ER and NR ionization yields will impact direct dark matter searches with LAr, extending the observation window to low-mass candidates, like weakly interacting massive particles of few $\mathrm{GeV} / \mathrm{c}^{2}$ mass, axionlike particles, dark photons, and sterile neutrinos, and to neutrino bursts from core-collapse supernovae [47]. Dedicated campaigns of measurement with setups exposed to neutron beams are highly desirable in the future to improve and better constrain response models at the $\mathrm{keV}$ scale.

\section{ACKNOWLEDGMENTS}

We are grateful to Francesc Salvat and Pascal Lablanquie for their comments and suggestions. The DarkSide Collaboration offers its profound gratitude to the Gran Sasso National Laboratory and its staff for their invaluable technical and logistical support. We also thank the Fermilab Particle Physics, Scientific, and Core Computing Divisions. Construction and operation of the DarkSide-50 detector was supported by the U.S. National Science Foundation (NSF) (Grants No. PHY-0919363, No. PHY-1004072, No. PHY-1004054, No. PHY-1242585, No. PHY1314483, No. PHY-1314501, No. PHY-1314507, No. PHY-1352795, No. PHY-1622415, and associated collaborative Grants No. PHY-1211308 and No. PHY1455351), the Italian Istituto Nazionale di Fisica Nucleare, the U.S. Department of Energy (Contracts No. DE-FG02-91ER40671, No. DEAC02-07CH11359, and No. DE-AC05-76RL01830), the Polish NCN (Grant No. UMO-2014/15/B/ST2/02561) and the Foundation for Polish Science (Grant No. Team2016-2/17). We also acknowledge financial support from the French Institut National de Physique Nucléaire et de Physique des Particules (IN2P3), the IN2P3-COPIN consortium (Grant No. 20-152), and the UnivEarthS LabEx program (Grants No. ANR-10-LABX-0023 and No. ANR-18-IDEX-0001), from the So Paulo Research Foundation (FAPESP) (Grant No. 2016/09084-0), from the Interdisciplinary Scientific and Educational School of Moscow University "Fundamental and Applied Space Research," from the Program of the Ministry of Education and Science of the Russian Federation for higher education establishments, Project No. FZWG-2020-0032 (2019-1569), and from IRAP AstroCeNT funded by FNP from ERDF. Isotopes used in this research were supplied by the United States Department of Energy Office of Science by the Isotope Program in the Office of Nuclear Physics.
[1] E. Aprile et al. (XENON Collaboration), Phys. Rev. Lett. 121, 111302 (2018).

[2] X. Cui et al. (PandaX-II Collaboration), Phys. Rev. Lett. 119, 181302 (2017).

[3] D. Akerib et al. (LUX Collaboration), Phys. Rev. Lett. 118, 021303 (2017).

[4] R. Ajaj et al. (DEAP Collaboration), Phys. Rev. D 100, 022004 (2019).

[5] P. Agnes et al. (DarkSide Collaboration), Phys. Rev. D 98 , 102006 (2018).

[6] E. Aprile et al. (XENON Collaboration), Phys. Rev. Lett. 123, 251801 (2019).

[7] E. Aprile et al. (XENON Collaboration), Phys. Rev. Lett. 123, 241803 (2019).
[8] P. Agnes et al. (DarkSide Collaboration), Phys. Rev. Lett. 121, 081307 (2018).

[9] P. Agnes et al. (DarkSide Collaboration), Phys. Rev. Lett. 121, 111303 (2018).

[10] P. Sorensen et al. (XENON10 Collaboration), Nucl. Instrum. Methods Phys. Res., Sect. A 601, 339 (2009).

[11] L. Goetzke, E. Aprile, M. Anthony, G. Plante, and M. Weber, Phys. Rev. D 96, 103007 (2017).

[12] D. Akerib et al. (LUX Collaboration), arXiv:1608.05381.

[13] E. Aprile et al. (XENON Collaboration), Phys. Rev. D 97, 092007 (2018).

[14] E. Aprile et al. (XENON Collaboration), Phys. Rev. D 99, 112009 (2019). 
[15] P. Agnes et al. (DarkSide Collaboration), Phys. Lett. B 743, 456 (2015).

[16] P. Agnes et al. (DarkSide Collaboration), Phys. Rev. D 93, 081101 (2016); 95, 069901(A) (2017).

[17] P. Agnes et al. (DarkSide Collaboration), J. Instrum. 11, P03016 (2016).

[18] P. Agnes et al. (DarkSide Collaboration), J. Instrum. 12, P10015 (2017).

[19] M.-M. Bé, V. Chisté, C. Dulieu, X. Mougeot, V. Chechev, F. Kondev, A. Nichols, X. Huang, and B. Wang, Table of Radionuclides, Monographie BIPM-5 Vol. 7 (Bureau International des Poids et Mesures, Pavillon de Breteuil, F-92310 Sèvres, France, 2013).

[20] X. Mougeot, Appl. Radiat. Isot. 134, 225 (2018).

[21] X. Mougeot, Appl. Radiat. Isot. 154, 108884 (2019).

[22] M. Wang, W. Huang, F. Kondev, G. Audi, and S. Naimi, Chin. Phys. C 45, 030003 (2021).

[23] D. E. Cullen, Program RELAX: A code designed to calculate atomic relaxation spectra of x-rays and electrons, Lawrence Livermore National Laboratory, Technical Report No. UCRL-ID.110438, 1992.

[24] D. E. Cullen, A survey of atomic binding energies for use in EPICS2017, IAEA, Technical Report No. IAEA-NDS0224, 2017.

[25] U. Fano, Phys. Rev. 72, 26 (1947).

[26] A. G. Santos-Ocampo and D. C. Conway, Phys. Rev. 120, 2196 (1960).

[27] P. W. Dougan, K. W. D. Ledingham, and R. W. P. Drever, Philos. Mag. A 7, 475 (1962).

[28] D. Totzek and K. W. Hoffmann, Z. Phys. 205, 137 (1967).

[29] T. Doke, A. Hitachi, S. Kubota, A. Nakamoto, and T. Takahashi, Nucl. Instrum. Methods 134, 353 (1976).

[30] J. Thomas and D. A. Imel, Phys. Rev. A 36, 614 (1987).
[31] M. Jaskolski and M. Wojcik, J. Phys. Chem. A 115, 4317 (2011).

[32] T. Doke, A. Hitachi, J. Kikuchi, K. Masuda, H. Okada, and E. Shibamura, Jpn. J. Appl. Phys. 41, 1538 (2002).

[33] P. Sorensen and C. E. Dahl, Phys. Rev. D 83, 063501 (2011).

[34] T. Doke, H. Crawford, A. Hitachi, J. Kikuchi, P. Lindstrom, K. Masuda, E. Shibamura, and T. Takahashi, Nucl. Instrum. Methods Phys. Res., Sect. A 269, 291 (1988).

[35] P. Agnes et al., Phys. Rev. D 97, 112005 (2018).

[36] F. Bezrukov, F. Kahlhoefer, and M. Lindner, Astropart. Phys. 35, 119 (2011).

[37] J. P. Biersack and J. F. Ziegler, in Ion Implantation Techniques, edited by H. Ryssel and H. Glawischnig (Springer, Berlin, Heidelberg, 1982), pp. 122-156.

[38] P. Agnes et al. (DarkSide Collaboration), J. Instrum. 12, T12004 (2017).

[39] H. Cao et al. (SCENE Collaboration), Phys. Rev. D 91, 092007 (2015).

[40] T. H. Joshi et al., Phys. Rev. Lett. 112, 171303 (2014).

[41] J. Liu, R. Carr, D. A. Dwyer, W. Q. Gu, G. S. Li, R. D. McKeown, X. Qian, R. H. M. Tsang, F. F. Wu, and C. Zhang, Nucl. Instrum. Methods Phys. Res., Sect. A 797, 260 (2015).

[42] R. B. Firestone, C. Baglin, and S. Y. F. Chu, Table of Isotopes: 1996 CD ROM Edition (Wiley, New York, NY, 1996).

[43] G. Moliere, Z. Naturforsch. A 2, 133 (1947), http://zfn.mpdl .mpg.de/data/Reihe_A/2/ZNA-1947-2a-0133.pdf.

[44] W. Lenz, Z. Phys. 77, 713 (1932).

[45] H. Jensen, Z. Phys. 77, 722 (1932).

[46] I. S. Tilinin, Phys. Rev. A 51, 3058 (1995).

[47] P. Agnes et al. (DarkSide 20k Collaboration), J. Cosmol. Astropart. Phys. 03 (2021) 043. 